\title{
Special Section Guest Editorial: Active Spectroscopy
}

\author{
Joseph Meola, ${ }^{\text {a }}$ Robert Bedford, ${ }^{\text {a }}$ Kristin Dewitt, ${ }^{\text {b }}$ Timothy J. Johnson, ${ }^{\text {c }}$ \\ and Christopher A. Kendziora ${ }^{d}$ \\ ${ }^{a}$ Air Force Research Laboratory, Wright-Patterson Air Force Base, Ohio, United States \\ ${ }^{\mathrm{b}}$ Intelligence Advanced Research Projects Activity, River Park, Maryland, United States \\ ${ }^{c}$ Pacific Northwest National Laboratory, Richland, Washington, United States \\ ${ }^{\mathrm{d}}$ Naval Research Laboratory, Washington, DC, United States
}

Passive hyperspectral imaging (HSI) has found widespread use in various applications, such as mineral mapping, search and rescue, vegetation health assessment, and target detection. However, recent advances in bright optical sources with both higher power and broader spectral coverage allow for their use as illumination sources for spectral receivers. The use of such nonthermal laser sources in recent years has brought to fruition active HSI methods, some of which are described in this special section. The flexibility afforded by control of an illumination source can extend operability of solar reflective systems for traditional missions as well as improve performance for certain applications, e.g. trace chemical detection, and even enable entirely new applications, such as 3D-HSI. However, numerous challenges still exist for the development and use of active spectral systems. These challenges include opto-mechanical designs, increased power and spectral range of sources, system trade-offs, calibration, data exploitation, coherence effects such as speckle, and atmospheric compensation. Equally important for development of useful exploitation methods is the understanding and modeling of material spectral properties and associated active spectral phenomenology. The eleven papers in this special section cover recent developments in active spectroscopy, including new phenomenology and applications, advances in systems and components, and new methods for data processing and exploitation.

Active spectroscopy can enable new domains of research-for example, Zhang et al. design and optimize a confocal dark-field ophthalmic probe to enable real-time noninvasive analysis of the aqueous humor in the eye by Raman spectroscopy. Dark-field illumination prevents the excitation light from directly reaching vulnerable retinal tissue. A functional contact probe prototype is developed and tested on ex vivo rabbit eyes.

Gas chromatography (GC) is a staple analytical technique used to separate chemical mixtures prior to identification, often combined with another technique such as mass spectrometry or Fourier transform infrared spectroscopy. Breshike et al. perform in situ infrared spectroscopy during the process of separating the analytes along the GC column by spin coating a stationary phase onto a germanium prism and actively probing the stationary phase in an attenuated total reflectance configuration with a quantum cascade laser. The GC column is formed by pressing a molded epoxy lid, with grooves that form the tubular column, onto the stationary phase coated prism.

Active spectroscopy can significantly extend the functionality and sensitivity beyond what can be accomplished using passive systems: For example, Goyal et al. develop a chemical detector that combines tunable quantum cascade laser illumination with a mercury cadmium telluride camera to capture hyperspectral images of the diffuse reflectance from a target surface in the longwave infrared. The system is able to generate individual hypercubes in $<0.1$ seconds, achieve areal coverage rates of $>60 \mathrm{~cm}^{2}$ per second by raster scanning, and detect target chemicals at standoff distances ranging from 0.1 to 25 meters. Practical detection demonstrations include explosive residues on a computer keyboard, residual pharmaceuticals inside a plastic bag, and a contaminated fingerprint on a cell phone case.

Ifarraguerri et al. describe an active infrared spectrometer for trace standoff chemical detection at a standoff of up to 15 meters. Active illumination with a continuous spectral range from 3.6 to $11 \mu \mathrm{m}$ is provided by a supercontinuum (SC) fiber laser source. In order to maximize

(C) 2020 Society of Photo-Optical Instrumentation Engineers (SPIE) 
energy transmission and capture given the fundamental and practical constraints imposed by the SC source, spectrometer, and detectors, an all-reflective, off-axis configuration is used for both the transmitter and receiver, with custom-designed enhanced gold surfaces. The spectrometer captures $>90 \%$ of the source energy and transmits it with low loss to a remote target while maintaining a nearly diffraction-limited Gaussian beam.

Pfeuffer et al. describe a field-portable fiber-laser based hyperspectral imaging system. The prototype uses a hybrid of frequency and time-resolved coherent anti-Stokes Raman scattering (CARS) spectroscopic techniques with spatial scanning for rapid detection of microscopic traces of explosives on liquid or solid surfaces. An all-fiber light source architecture enables the three CARS excitation beams to be generated from a compact, single-unit femtosecond fiber laser coupled to a highly nonlinear optical fiber.

Galán-Freyle et al. demonstrate standoff detection of traces of the explosive RDX by laserinduced thermal emission (LITE). Traditional $\mathrm{CO}_{2}$ laser based LITE requires careful alignment, is susceptible to $\mathrm{CO}_{2}$ signal interference, and suffers from power density loss at the sample with increased standoff distance. The paper describes a remote chopped-laser induction system for LITE detection using a $\mathrm{CO}_{2}$ laser source coupled to a focusing telescope that was built to solve some of these limitations. Colón-Mercado et al. report using mid-infrared (MIR) laser spectroscopy measurements coupled with multivariate analysis (MVA) to detect and classify high explosives on various substrates. A mid-infrared quantum cascade laser is coupled to a grazing angle probe mount to illuminate the surface at an angle of 82 degrees with respect to surface normal. Diffuse backscattered radiation is measured to accurately identify traces of high explosives with a low false alarm rate.

Breshike et al. demonstrate detection of trace explosives on surfaces at standoff of a few meters by sweeping the wavelength of an infrared quantum cascade laser for illumination collecting backscattered signal onto an infrared focal plane array. Hyperspectral image cubes containing both spatial and spectral information are compared against a known threat library using a convolutional neural network $(\mathrm{CNN})$ trained on purely synthetic data. The CNN identifies chemicals by their IR signature and identifies their location within the image.

It is important to be able to leverage new modeling and simulation approaches to address active spectroscopy challenges. For example, the complex refractive index, $\hat{n}=n+\mathrm{i} k$, can be used to simulate infrared spectra, accounting for reflection, refraction, and absorption phenomena as a function of wavelength. Bernacki et al. use experimentally measured $n / k$ vectors for solid and liquid analytes to predict thin film spectra on substrates including both conductors and dielectrics. Comparison of the calculated spectra with experimental hemispherical reflectance data shows excellent agreement, indicating promise for the use of measured $n / k$ data to synthesize reference spectral data.

Active standoff detection of hazardous materials using infrared backscattering spectroscopy shows promise due to its speed of detection, sensitivity, chemical specificity, eye safety, and the ability to perform covert detection. However, the experienced practitioner knows that infrared diffuse reflectance spectra of trace particles on substrates exhibit a strong dependence on substrate type, particle size, and mass loading, which can dramatically alter spectral signatures. Furstenburg et al. have developed models to calculate the diffuse reflectance spectra of trace particles on both relatively smooth substrates and very rough substrates. The primary inputs to the models are the analyte and substrate optical constants as a function of wavelength, the particle size distribution, and average mass loading. The models were developed and their accuracies validated by comparing to experimentally measured diffuse reflectance spectra of several carefully prepared samples.

Trace chemical detection and classification in standoff reflection-based spectroscopic data is challenging due to the variability of measured data and the lack of physics-based models that can accurately predict spectra. To simplify calculations, most available models assume that the chemical takes the form of spherical particles or perfectly uniform thin films. Murphy et al. present a signature model, called the sparse transfer matrix, that is designed for more realistic chemical presentations, namely of a non-uniform chemical film.

The guest editors wish to thank the Optical Engineering editorial team, the authors, and reviewers for their effort to present this section. Our goal with this special section is to inspire continued research and advances in the field of active spectroscopy. 